This is an electronic reprint of the original article. This reprint may differ from the original in pagination and typographic detail.

Author(s): Lehto, Joni; Alén, Raimo; Kleen, Marjatta

Title: $\quad$ Sulfur-free pulping of hot-water-extracted spruce sawdust

Year: $\quad 2016$

Version:

Please cite the original version:

Lehto, J., Alén, R., \& Kleen, M. (2016). Sulfur-free pulping of hot-water-extracted spruce sawdust. Nordic Pulp and Paper Research Journal, 31(1), 41-48.

https://doi.org/10.3183/npprj-2016-31-01-p041-048

All material supplied via JYX is protected by copyright and other intellectual property rights, and duplication or sale of all or part of any of the repository collections is not permitted, except that material may be duplicated by you for your research use or educational purposes in electronic or print form. You must obtain permission for any other use. Electronic or print copies may not be offered, whether for sale or otherwise to anyone who is not an authorised user. 


\section{Sulfur-free pulping of hot-water-extracted spruce sawdust}

Joni Lehto, Raimo Alén, and Marjatta Kleen

KEYWORDS: Hot-water pre-treatment, Norway spruce, Aliphatic acids, Hemicelluloses, Carbohydrates, Lignin, Extractives, Molar-mass distribution

SUMMARY: Softwood (Picea abies) sawdust was hotwater-extracted with two multi-phase processes (160$180^{\circ} \mathrm{C}, 100-280 \mathrm{~min}$, and liquor-to-wood ratio $5 \mathrm{l} / \mathrm{kg}$ ) corresponding to P-factors of $\sim 780$ (yield $80.0 \%$ ) and $\sim 4580$ (yield $72.4 \%$ ). The pre-treated materials were then pulped with the soda-AQ cooking $\left(170^{\circ} \mathrm{C}, \mathrm{NaOH}\right.$ charge $20 \%$, AQ charge $0.1 \%, 30-150 \mathrm{~min}$, and liquor-to-wood ratio $51 / \mathrm{kg}$ ), resulting in a yield range of $36.1-61.4 \%$ (for reference cooks without pre-extraction $47.9-60.0 \%$ ). It was observed that the pre-extraction under moderate conditions enhanced the dissolution especially of largemolar-mass lignin during cooking, and the total amount of the dissolved lignin was also higher than that removed from the material pre-extracted under harsh conditions. Additionally, in the former case the pulps with rather good properties (high yield and low amount of pulp rejects) were obtained, whereas in the latter case, a pronounced degradation of pulp carbohydrates to aliphatic acids was observed resulting in lower pulp properties.

\section{ADDRESSES OF THE AUTHORS:}

Joni Lehto (joni.t.lehto@jyu.fi) and Raimo Alén (raimo.j.alen@jyu.fi): Laboratory of Applied Chemistry, Department of Chemistry, Survontie 9, FI-40014 University of Jyväskylä, Finland. Marjatta Kleen (marjattakleen@hotmail.com): VTT Technical Research Centre of Finland Ltd, Espoo, Finland

Corresponding author: Joni Lehto

Chemical pulp mills use large quantities of lignocellulosic feedstocks to produce cellulosic fiber. However, the chemical delignification process is rather unselective, and significant part of raw wood material is dissolved into the cooking liquor ("black liquor", BL), which is burned in the recovery furnace for recovering cooking chemicals and producing energy (Adams 1997; Vakkilainen 2008). The dissolved organic fraction in BL contains, in addition to carbohydrates-derived degradation products (aliphatic carboxylic acids), degradation fractions of lignin, together with minor amounts of extractives (Niemelä et al. 1985; Niemelä, Sjöström 1986a, 1986b, 1986c; Niemelä 1988a, 1988b; 1989; 1990a, 1990b; Käkölä et al. 2007, 2008; Pakkanen et al. 2012; Pakkanen, Alén 2012, 2013). Due to the low heating value of carbohydrates-derived materials, compared to those of lignin, the partial removal of these constituents by different pre-treatment stages prior to the main pulping stage is attractive (Al-Dajani, Tschirner 2008).

The conversion of traditional pulp mills into integrated forest biorefineries (IFBRs) has gained a significant attention during the past decades, and one of the most promising approaches towards a full-scale IFBR process includes the incorporation of a pre-treatment stage of wood prior to alkaline pulping (van Heiningen 2006; Carvalheiro et al. 2008; Baijpai 2012). Several pretreatment methods can be utilized for producing carbohydrates- and lignin-containing fragments from wood. These side-stream fractions can be further converted into desired chemicals, other biomaterials, or biofuels, thus enhancing the overall efficiency of the wood utilization and also improving the profitability of producing wood-based cellulosic fiber (Kamm, Kamm 2006; Kamm et al. 2006; Sánchez, Cardona 2008). By using different pre-treatments, it is also possible to increase the reactivity of feedstock material, leading to potential savings in the subsequent pulping operations (Hendriks, Zeeman 2009; Kumar et al. 2009). One of the most studied pre-treatment methods includes pressurized hot-water extraction (PHWE) (Smith 2002; Amidon, Liu 2009).

PHWE (often also known as sub-critical water extraction, superheated water extraction, extraction with water at elevated temperatures and pressures, or near critical water extraction) has become a potential green extraction method for several compounds present in many different biological matrices (Smith 2002; Wiboonsirikul, Adachi 2008; Amidon, Liu 2009). PHWE is typically performed at the temperatures above $100^{\circ} \mathrm{C}$ and below $374^{\circ} \mathrm{C}$ (the critical temperature of water). The enhancement on the extraction efficiency of PHWE can be attributed to improvement in the solubility and mass transfer effects, and to increased disruption of surface equilibria (Teo et al. 2010; Penttilä et al. 2013). Due to the basic phenomena occurring during the process, PHWE performed with lignocellulosic materials (LCMs) is called autohydrolysis (Carvalheiro et al. 2008). During the first part of autohydrolysis, catalytical hydronium ions come solely from the water autoionization reactions (Garrote et al. 2001; Chen et al. 2010; Schild et al. 2010). Hydronium ions act as a catalyst, especially during the early hydrolysis reactions of LCMs, leading to selective cleavage of the glycosidic linkages and in particular, the acetyl groups of hemicelluloses.

In the second part of autohydrolysis, hydronium ions coming from acetic and possibly from uronic acids start acting as catalysts (Garrote et al. 1999a, 1999b; Carvalheiro et al. 2008). In the latter part of this process, the contribution of hydronium ions originating from organic acids is higher than that from water autoionization, resulting in the hydrolysis of the linkages between hemicelluloses and lignin, as well as the glycolsidic bonds of carbohydrates. Autohydrolysis technology covers a wide range of treatments, including water- and steam-based processes. On an industrial scale, autohydrolysis is applied in the pre-hydrolysis kraft 
(PHK) process for producing dissolving pulps (Sixta et al. 2006; Sixta, Schild 2009; Schild et al. 2010).

The main aim of this study was to clarify the effects of various PHWE conditions on the sulfur-free pulping of Norway spruce (Picea abies) sawdust. This approach included two PHWE processes performed with very different intensities followed by soda-AQ pulping conducted under typical pulping conditions. In addition, the detailed compositions of the resulting side streams (BLs), especially concentrating on the dissolved lignin and carbohydrates-derived carboxylic acids, were determined.

\section{Materials and Methods}

\section{Pre-treatments}

The pilot-scale extractions were performed by extracting non-dried, screened industrial (sawmill) spruce (Picea abies) sawdust with deionized water in a purpose-built 301 vessel (VTT, Espoo, Finland). The extraction apparatus contained 5 separately controlled vessels for pre-heating of different solvents or pre-heating to different temperatures, to enable fast and accurate delivery of the extraction solvent. Thus, the pre-contact between the up-warming water and the material to be extracted was eliminated. In addition, the pilot-scale apparatus used circulation of the extraction liquid (i.e., hot water) during the extraction. The pilot-scale PHWE was performed with two intensities. PHWE 1 was performed as two stages; first stage at $160^{\circ} \mathrm{C}$ for $40 \mathrm{~min}$ (P-factor $\sim 181$, hydrolysate $\mathrm{pH} 3.67$ ) and the second stage at $170^{\circ} \mathrm{C}$ for $60 \mathrm{~min}$ (P-factor $\sim 597$, hydrolysate $\mathrm{pH}$ 3.45, combined total P-factor 780) (Brasch, Free 1965; Carvalheiro et al. 2008; Tunc, van Heiningen 2009) liquid-to-wood ratio (L/W) being $5 \mathrm{l} / \mathrm{kg}$ in both cases. Between these two stages, the hydrolysate was drained off from the reactor as completely as possible. After both extraction steps, extracted material was washed for $30 \mathrm{~min}$ with water $\left(50^{\circ} \mathrm{C}, \mathrm{L} / \mathrm{W} 5 \mathrm{l} / \mathrm{kg}\right)$, removed from the reactor, and stored in a freezer. PHWE 2 was performed as three stages: the first stage at $160^{\circ} \mathrm{C}$ for $40 \mathrm{~min}$ (hydrolysate $\mathrm{pH} 3.62$ ), the second stage at $170^{\circ} \mathrm{C}$ for

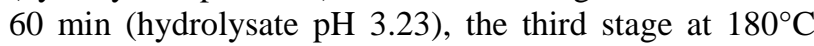

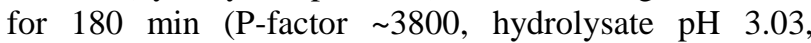
combined P-factor 4580 ) ( $\mathrm{L} / \mathrm{W}$ was $51 / \mathrm{kg}$ in all cases). Between each stage, the extract was drained from the reactor. Finally, the extracted material was washed and stored as previously mentioned.

\section{Analyses of feedstock materials for pulping experiments}

For the chemical analyses (i.e., content of carbohydrates, lignin, and extractives), the reference and extracted sawdust samples were stored as such in a freezer, and their moisture content was determined. In all wetchemistry analyses, the results were an average of two parallel determinations and calculated as a percentage of dry sample. The extractives content of sawdust was determined by extracting the sample with acetone (from Sigma-Aldrich) for $4 \mathrm{~h}$ in a Soxhlet apparatus (TAPPI Test Methods T280 pm-99). The extract obtained was dried before weighing by vacuum evaporation with a rotary evaporator (Heidolph VV2000) and was finally accomplished by means of a gentle nitrogen gas stream.

The lignin content of the extractives-free sawdust sample (about $200 \mathrm{mg}$ ) was determined as the sum of "acid-insoluble Klason lignin" and "acid-soluble lignin" (TAPPI Test Methods T222 om-98 and T249 cm-00). In this determination, the method employed was that sawdust was first treated with $\mathrm{H}_{2} \mathrm{SO}_{4}$ (from SigmaAldrich), and the lignin precipitated was filtered off, washed, dried, and weighed. The content of acid-soluble lignin was determined using a Beckman DU 640 UV/Visspectrophotometer at $205 \mathrm{~nm}$ (to avoid the interfering influence of furanoic compounds) after dilution of one portion of the hydrolysate with the corresponding aqueous solution of $\mathrm{H}_{2} \mathrm{SO}_{4}(0.5 \%)$ until the absorbance (A) was in the range 0.3 to 0.8 . The concentration of dissolved lignin $(C, \mathrm{~g} / \mathrm{l})$ was calculated according to $E q 1$.

$$
C=\frac{A}{a * b}
$$

where $a$ is absorptivity $(110 \mathrm{l} /(\mathrm{gcm}))($ Swan 1965$)$ and $b$ is light path $(\mathrm{cm})$.

The content of different monosaccharides (i.e., arabinose, galactose, glucose, mannose, and xylose) in the Klason hydrolysates was determined by means of a Dionex high performance liquid chromatography-pulse amperometric detection (HPLC-PAD) equipped with an AS50 autosampler, a LC25 chromatography oven, a GS50 gradient pump, a CarboPac PA-1 column, and an ED50 detector with carbohydrate pulsing. Samples were eluted (ultra high quality (UHQ) water with $\mathrm{NaOH}$ (from Merck) gradient) at a flow rate of $0.3 \mathrm{ml} / \mathrm{min}$. The UHQ water used for the preparation of the mobile phase was degassed via ultrasonic treatment for approximately 15 min prior to use. Post-column alkali $(\mathrm{NaOH})$ addition was performed at a flow rate of $0.1 \mathrm{ml} / \mathrm{min}$ with an IC25 isocratic pump to enhance the performance of PAD. Data were stored and processed using a Dionex Chromeleon (6.50) data system. The peak identification and the massbased response factors between the internal standard (fucose from Sigma) and each monosaccharide were based on separate runs with model monosaccharides (all from Fluka).

\section{Pulping experiments}

The soda-AQ cooking experiments were carried out in a laboratory-scale, oil-heated batch digester (CRS CAS 420) equipped with $1.25-1$ rotating stainless steel autoclaves. The cooking conditions were as follows: alkali $(\mathrm{NaOH})$ charge $20 \%$ based on oven dried (o.d.) feedstock; AQ charge $0.1 \%$ based on o.d. feedstock; cooking times 30, 60, 90, 120, and $150 \mathrm{~min}$; cooking temperature $170^{\circ} \mathrm{C}$; and $\mathrm{L} / \mathrm{W}$ ratio $51 / \mathrm{kg}$. At the end of each cook, the autoclaves were removed from the oil bath and cooled rapidly with cold tap water. The BL was separated from the pulp by pressing and stored in a freezer for further analyses, including the determination of volatile acids, hydroxy acids, and lignin.

\section{Analysis of the BLs}

The content of dissolved lignin in the BLs was determined by using a Beckman DU 640 UV/Visspectrophotometer at $280 \mathrm{~nm}$ after dilution of one portion 
of the $\mathrm{BL}$ with $0.1 \mathrm{M} \mathrm{NaOH}$ solution until the absorbance (A) was in the range 0.3 to 0.8 . The concentration of dissolved lignin $(C, \mathrm{~g} / \mathrm{l})$ was calculated according to $E q 1$. In this case, the absorptivity value used was $21.61 /(\mathrm{gcm})$ (Alén, Hartus 1988).

The molar-mass distributions (MMDs) of the dissolved lignin were determined by GPC using a Waters high performance liquid chromatography (HPLC) system equipped with a column (460 mm x $10 \mathrm{~mm}$ i.d.) filled with Superdex 75 gel (Pharmacia) (Pakkanen, Alén 2012b; Lehto et al. 2015). Samples were diluted before measurement with the eluent $(0.1 \mathrm{M} \mathrm{NaOH})$ and filtered with a nylon syringe filter $(0.45 \mu \mathrm{m})$. The flow rate of the eluent in HPLC was $0.3 \mathrm{ml} / \mathrm{min}$ at room temperature. Detection was performed using a Waters 996 photodiode array (PDA) detector within the wavelength range $240 \mathrm{~nm}$ to $400 \mathrm{~nm}$, and a wavelength of $280 \mathrm{~nm}$ was used for the determination of relative molar masses. Calibration of the GPC system was performed with a commercial set of protein standards (Sigma-Aldrich) and a number of lignin-like monomer/oligomer model compounds (Ristolainen et al. 1996).

Volatile acids (i.e., formic and acetic acids) were determined as their benzyl ester derivatives by means of the previously described method (Alén et al. 1985) using an Agilent 6850 gas chromatograph with flame-ionization detector (GC-FID) equipped with a capillary column Agilent HP-5 (30 m x $0.32 \mathrm{~mm}$ i.d., film thickness $0.25 \mu \mathrm{m})$. Nitrogen was used as a carrier gas, and crotonic acid ( $1 \mathrm{mg} / \mathrm{ml}$, from Fluka) was used as an internal standard. The temperature of both injection port and detector was $280^{\circ} \mathrm{C}$, and FID was operated with a hydrogen and air flow of $40 \mathrm{ml} / \mathrm{min}$ and $450 \mathrm{ml} / \mathrm{min}$, respectively. The column temperature program was $3 \mathrm{~min}$ at $60^{\circ} \mathrm{C}, 3^{\circ} \mathrm{C} / \mathrm{min}$ to $150^{\circ} \mathrm{C}, 15^{\circ} \mathrm{C} / \mathrm{min}$ to $230^{\circ} \mathrm{C}$, and $5 \mathrm{~min}$ at $230^{\circ} \mathrm{C}$. The total run time was approximately $44 \mathrm{~min}$. For GC-FID, a liquor sample of $1 \mathrm{ml}(1: 10$ dilution) and an internal standard of $0.5 \mathrm{ml}$ UHQ water solution were mixed and passed through a column filled with ion exchange resin (about $10 \mathrm{ml}$, Dowex 50x8, 50100 mesh, $\mathrm{H}^{+}$-form from Fluka). The column was washed with UHQ water to obtain a sample volume of 30-40 ml. $\mathrm{pH}$ of the samples was adjusted with tetra- $n$ butylammonium hydroxide (1.6 g/100 ml UHQ water, from Fluka) to a $\mathrm{pH}$ value of $\sim 8$, and samples were evaporated to dryness under a reduced pressure at about $35^{\circ} \mathrm{C}$. Samples were derivatized by adding a mixture of benzyl bromide (from Fluka) and acetone (in 1:20 ratio) and by shaking the samples for two hours at room temperature.

Hydroxy monocarboxylic and dicarboxylic acids were determined using an Agilent 6850 Series GC equipped with an HP-5 column (30 m x $0.32 \mathrm{~mm}$ i.d. with $0.25 \mu \mathrm{m}$ film thickness) and a FID (Alén et al. 1984; Käkölä et al. 2008). FID was operated at $300^{\circ} \mathrm{C}$ with a hydrogen and air flow of $40.0 \mathrm{ml} / \mathrm{min}$ and $450.0 \mathrm{ml} / \mathrm{min}$, respectively. The column temperature program was $5 \mathrm{~min}$ at $60^{\circ} \mathrm{C}$, $2^{\circ} \mathrm{C} / \mathrm{min}$ to $200^{\circ} \mathrm{C}, 70^{\circ} \mathrm{C} / \mathrm{min}$ to $290^{\circ} \mathrm{C}$, and $15 \mathrm{~min}$ at $290^{\circ} \mathrm{C}$. The total run time was approximately $91 \mathrm{~min}$. For GC-FID, a liquor sample of $1 \mathrm{ml}$ and an internal standard (xylitol from Fluka) of $1 \mathrm{ml}$ UHQ water solution (concentration $0.1 \mathrm{mg} / \mathrm{ml}$ ) were mixed and passed through a column filled with weakly acidic cation exchange resin (about $10 \mathrm{ml}$, Amberlite IRC-50, 1650 mesh, $\mathrm{NH}_{4}{ }^{+}$-form, from Fluka). The column was washed with UHQ water to obtain a sample volume of 30-40 ml, and this aqueous sample was then evaporated to dryness under reduced pressure at about $35^{\circ} \mathrm{C}$. Finally, the residue was per(trimethylsilyl)ated by adding $1 \mathrm{ml}$ pyridine (from VWR Prolabo) and $0.5 \mathrm{ml} \mathrm{N,O}$ bis(trimethylsilyl)trifluoroacetamide (BSTFA, from Regisil) containing $1 \%$ trimethylchlorosilane (TMCS, from Regisil), and the mixture was shaken for approximately $60 \mathrm{~min}$ at room temperature. The identification of the individual acid derivatives was made using the same GC system, which was equipped with a mass selective detector (GC-MSD). The interpretation of the mass spectra was based on data from previous studies and performed by an HP ChemStation (A06.03) chromatography data system. For the quantitative calculations, the mass-based response factors between xylitol (1.00) and the peaks derived from the acids studied were based on the data given by Alén et al. (1984).

\section{Results and discussion}

\section{Feedstock materials and pre-treatments}

The chemical compositions of the sawdust samples before and after PHWE are presented in Table 1. The yields of the pre-treatments were $80.0 \%$ and $72.4 \%$ of o.d. feedstock for PHWE 1 (low intensity pre-treatment) and PHWE 2 (high intensity pre-treatment), respectively. The effects of the pre-treatments were clearly visible, especially on the decreasing content of the hemicelluloses-derived carbohydrates. Especially, during the harsh PHWE 2 pre-treatment, practically all (up to $85 \%$ of the original) hemicelluloses were dissolved, the insoluble fraction consisting mainly of glucose (most likely originating from cellulosic fibers) and lignin. Corresponding reduction of hemicelluloses in the case of PHWE 1 was roughly $50 \%$ of the original. Of lignin, PHWE 1 and PHWE 2 pre-treatments removed roughly $6 \%$ and $12 \%$ of the total initial lignin, respectively.

\section{Pulping experiments}

The yield data on soda-AQ pulping experiments conducted with the reference (i.e., without hot-water extraction) and hot-water-extracted spruce sawdust samples are presented in Table 2. The effect of PHWE on pulping performance was clear, as distinctly lower total pulp yields were achieved when the pre-treated material was cooked. Slightly higher overall yields, compared to those from the sawdust treated with moderate autohydrolysis (PHWE 1), were achieved with sawdust pre-treated under harsh pre-treatment conditions (PHWE 2 ). However, when the cooking yields were calculated as $\%$ of the material loaded into the reactors (i.e., material removed during the autohydrolysis was not taken into consideration), the yields were clearly the highest for harshly treated sawdust (PHWE 2).

The data presenting the amount of pulp rejects (i.e., material not passing through a $0.2-\mathrm{mm}$ screen) after each cooking experiment are shown in Table 3. The highest pulp rejects were determined for the pulps from PHWE 2 
BIOREFINERY

Nordic Pulp \& Paper Research Journal Vol 31 no (1) 2016

Table 1 - Chemical composition of the untreated (reference) and PHWE sawdust samples

\begin{tabular}{lccc}
\hline $\begin{array}{l}\text { Component (\% o.d. } \\
\text { feedstock) }\end{array}$ & Reference & PHWE 1 & PHWE 2 \\
\hline Monosaccharides* & 58.1 & 60.4 & 58.2 \\
Arabinose & 1.0 & $<0.1$ & $<0.1$ \\
Galactose & 1.6 & 0.4 & $<0.1$ \\
Glucose & 39.7 & 51.6 & 55.4 \\
Mannose & 10.7 & 4.8 & 0.9 \\
Xylose & 5.0 & 3.7 & 1.9 \\
Lignin & 29.8 & 35.2 & 36.6 \\
Klason & 29.4 & 34.9 & 36.4 \\
Acid soluble & 0.4 & 0.3 & 0.2 \\
ASMs* ("extractives") & 1.3 & 2.6 & 4.0 \\
Others & 10.8 & 1.8 & 1.2 \\
\hline
\end{tabular}

${ }^{*}$ Monosaccharide moieties are presented as anhydro sugars.

${ }^{* *}$ Acetone-soluble materials.

Table 2 - Yield of the soda-AQ pulping experiments

\begin{tabular}{|c|c|c|c|c|c|c|c|c|c|c|}
\hline Time/min & \multicolumn{2}{|c|}{30} & \multicolumn{2}{|c|}{60} & \multicolumn{2}{|c|}{90} & \multicolumn{2}{|c|}{120} & \multicolumn{2}{|c|}{150} \\
\hline Sample & $C Y^{*}$ & $\mathrm{TCY}^{* *}$ & $C Y^{*}$ & $\mathrm{TCY}^{* *}$ & $C Y^{*}$ & $\mathrm{TCY}^{* *}$ & $C Y^{*}$ & $\mathrm{TCY}^{* *}$ & $C Y^{*}$ & $\mathrm{TCY}^{* *}$ \\
\hline Reference & 60.0 & - & 53.1 & - & 50.2 & - & 49.3 & - & 47.9 & - \\
\hline PHWE 1 & 61.2 & 48.9 & 54.2 & 43.4 & 49.9 & 39.9 & 47.5 & 38.0 & 45.1 & 36.1 \\
\hline PHWE 2 & 64.9 & 46.7 & 61.4 & 44.2 & 60.0 & 42.5 & 54.8 & 39.5 & 53.2 & 38.3 \\
\hline
\end{tabular}

${ }^{*} \mathrm{CY}$ refers to cooking yield (\% of material charged into reactors).

${ }^{* *}$ TCY refers to total cooking yield ( $\%$ of original dry feedstock material before pre-treatment).

Table 3 - Amount of pulp reject (i.e., material not passing through a $0.2-\mathrm{mm}$ screen) calculated as $\%$ of cooking yield

\begin{tabular}{lccccc}
\hline Time/min & 30 & 60 & 90 & 120 & 150 \\
\hline Reference & 71.9 & 22.9 & 3.1 & 0.6 & 0.2 \\
PHWE 1 & 73.5 & 58.7 & 36.6 & 22.4 & 6.7 \\
PHWE 2 & 64.3 & 64.1 & 58.2 & 61.1 & 61.0 \\
\hline
\end{tabular}

material (with the harshest hot-water extraction). In these pulps, generally over $60 \%$ of the produced pulp consisted of rejected material, suggesting a very poor fibrillation performance. Pulping efficiency could not be enhanced by prolonging the cooking time. The similar, but smaller effect (i.e., hindered pulping performance due to the pretreatment) was discovered for PHWE 1 materials (less severe hot-water extraction). These results (together with the analysis of feedstock compositions and pulping yields) indicated some profound changes in the structure of wood matrix, most likely in the composition of lignin. Possible explanation could include, for example, various condensation reactions taking place during pretreatments, which rendered lignin structure insoluble and very resistant towards alkaline (but sulfur-free) cooking. This explanation was supported by previous studies (Leschinsky et al. 2008; 2009; Li, Gellerstedt 2008; Timilsena et al. 2013; Zhu et al. 2015), in which a decreased delignification after harsh auto-hydrolysis conditions was observed. In these studies, it was also reported that the maximum extractability of lignin could be achieved within a very narrow range of reaction time, and a significant decrease in lignin solubility and reactivity was reported after harsh auto-hydrolysis conditions. The decreased reactivity and solubility were explained by undesired re-polymerization reactions taking place during prolonged autohydrolysis
Table 4 - Polydispersity $\left(\overline{M_{w}} / \overline{M_{n}}\right)$ of lignin in BLs from the soda-AQ cooking of untreated (Reference) and pre-treated (PHWE) spruce sawdust

\begin{tabular}{lccccc}
\hline Time/min & $\mathbf{3 0}$ & $\mathbf{6 0}$ & $\mathbf{9 0}$ & $\mathbf{1 2 0}$ & $\mathbf{1 5 0}$ \\
\hline Reference & 10.5 & 11.9 & 11.0 & 10.7 & 9.4 \\
PHWE 1 & 10.4 & 11.5 & 11.1 & 10.9 & 10.8 \\
PHWE 2 & 7.5 & 8.0 & 8.8 & 9.2 & 9.7 \\
\hline
\end{tabular}

conditions. For example, it has been proposed that under mild acidic conditions, resonance stabilized carbonium ions are formed by the proton-induced elimination of water from the benzylic position (Chua, Wayman 1979; Li, Gellerstedt 2008; Borrega et al. 2011; Santos et al. 2013). In the $\beta-O-4$ structures, carbonium ions react further with $\beta$-ether linkages, leading to the cleavage of these linkages with the simultaneous formation of various intermediates, such as Hibbert's ketones. In addition, as strong electrophiles carbonium ions have the ability to participate in substitution reactions resulting in the formation of carbon-carbon bonds, and thus, in the repolymerization of dissolved lignin.

On the other hand, it has been suggested that under mild acidic conditions at elevated temperatures homolytic cleavage of the $\beta-O-4$ structures may occur leading into the formation of intermediate quinone methides (Leschinsky et al. 2008, 2009; Li, Gellerstedt 2008; Santos et al. 2013). These intermediates can then further undergo radical-exchange and radical-coupling reactions, the latter being able to form $\beta-\beta-, \beta-1-$, and $\beta-5$-type linkages. These structures are then condensed on the wood and equipment surfaces, thus impairing the subsequent delignification process. It has been shown in previous studies (Tsutsumi et al. 1995; Timilsena et al. 2013) that also the type of the lignin has an effect on the formation of re-condensed lignin structures. In general, 
the tendency to form these structures is more pronounced with guaiacyl-type lignin (i.e., softwood-type lignin) than with the corresponding syringyl lignin. In addition, the materials with lower content of guaiacyl-type subunits are delignified easier than their guaiacyl-rich counterparts.

\section{Analysis of the black liquor properties}

Lignin: The content of dissolved lignin in various sodaAQ BLs is presented in Fig 1. The highest lignin content was determined from the black liquors originating from cooking of wood from which $20 \%$ of initial material was removed during the hot-water extraction (PHWE 1). This finding suggested better solubility and enhanced diffusion of lignin out from the moderately pre-treated wood matrix, when compared to the reference and PHWE 2 materials. Explanations for the enhanced delignification in the case of PHWE 1 could include, for example, partial lignin depolymerization already during the autohydrolysis and more open structure of the pretreated wood matrix allowing the enhanced diffusion of the solubilized lignin out from the wood. In addition to the applied pre-treatment, prolonging the cooking time clearly seemed to enhance the lignin dissolution. On the other hand, the lowest lignin content was determined from the black liquors, which were cooked from severely extracted wood (PHWE 2). As discussed above, enhanced delignification took place within a very narrow range of pre-treatment time, and significant decreases in lignin solubility and reactivity after harsh autohydrolysis conditions have been reported (Leschinsky et al. 2008, 2009; Li, Gellerstedt 2008; Timilsena et al. 2013; Zhu et al. 2015). These observations were also in agreement with this study, as highly prolonged PHWE seemed to alter the structure of lignin in such a way that its dissolution was clearly hindered during the subsequent alkaline pulping.

$\overline{M_{w}}$ values of dissolved lignin in BLs from the soda-AQ cooking of spruce sawdust are presented in Fig 2. Again, the moderate PHWE seemed to facilitate the dissolution of lignin (i.e., larger-MM lignin was dissolved) from the pre-treated wood when compared to the reference and PHWE 2 materials. The MMs of the PHWE 1 lignin varied from $7,520 \mathrm{Da}$ to $6,830 \mathrm{Da}$, whereas the corresponding values for reference cooks covered the $\mathrm{MM}$ range from $6,080 \mathrm{Da}$ to $5,550 \mathrm{Da}$, suggesting improved diffusion of large-MM lignin out from the pretreated (more porous and open vascular structure) wood. In addition, the behavior of the dissolved lignin was found to be quite similar between these two cooking sets; i.e., prolonging the cooking time seemed to degrade the dissolved lignin further, which was seen as slightly decreasing MMs.

The lignin dissolved from PHWE 2 samples was very different when compared to the reference and PHWE 1 samples. In most cases, the MM of PHWE 2 lignin was the lowest of the three samples, ranging from 4,420 Da to 6,200 Da. In addition, opposite to the other samples, the MM of the dissolved lignin in PHWE 2 samples seemed to increase as the cooking time was elongated. It was evident that the structure of the lignin in the PHWE 2 feedstock was altered during the hot-water extraction in such a way that rather drastic alkaline cooking conditions were needed to dissolve it.

MMDs of the dissolved lignin from soda-AQ BLs after the cooking experiments conducted with different materials are presented in Fig 3. When compared to the reference samples, higher-MM lignin was dissolved rather readily (i.e., with short cooking times) from the moderately hot-water pre-treated samples, suggesting changes in the wood matrix caused by the pre-treatment (i.e., increasing porosity and enhanced diffusion), which allowed the large-MM lignin to dissolve out from the wood. In addition, prolonging the cooking time clearly degraded the dissolved lignin further in both cases. On the contrary, similar behavior could not be observed in the case of cooking the severely pre-treated sawdust. Clearly prolonged cooking times were needed for the solubilization of large-MM lignin from the PHWE 2 material.

Volatile and hydroxy acids: The contents of volatile and hydroxy acids in BLs are presented in Fig 4 and 5, respectively. Acetic acid content was clearly lower in those BLs cooked from harshly pre-treated sawdust, suggesting extensive removal of acetyl groups already during the pre-treatment stage. The effect of pretreatments on the content of formic acid was seemed to be twofold; mild pre-treatment conditions (PHWE 1) increased the formic acid content only slightly compared to reference cooks, whereas when applying harsh pretreatment conditions (PHWE 2), formic acid content

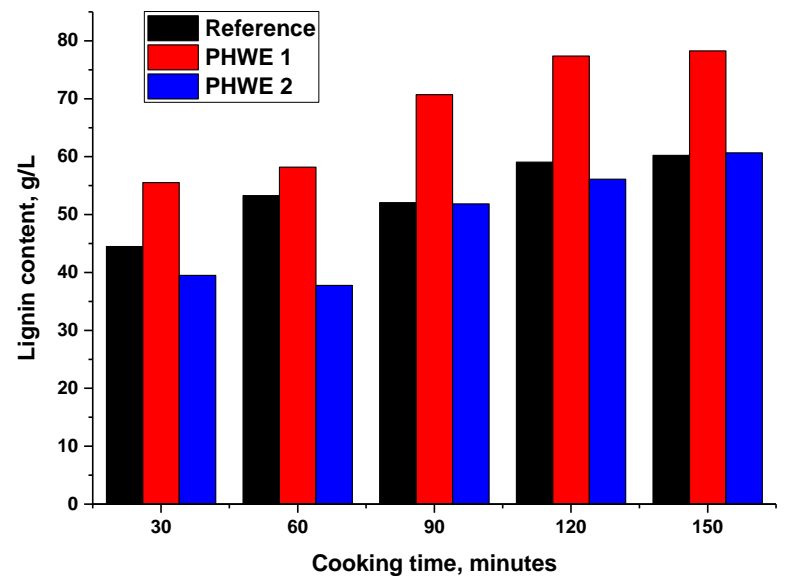

Fig 1 - Lignin content in various soda-AQ BLs.

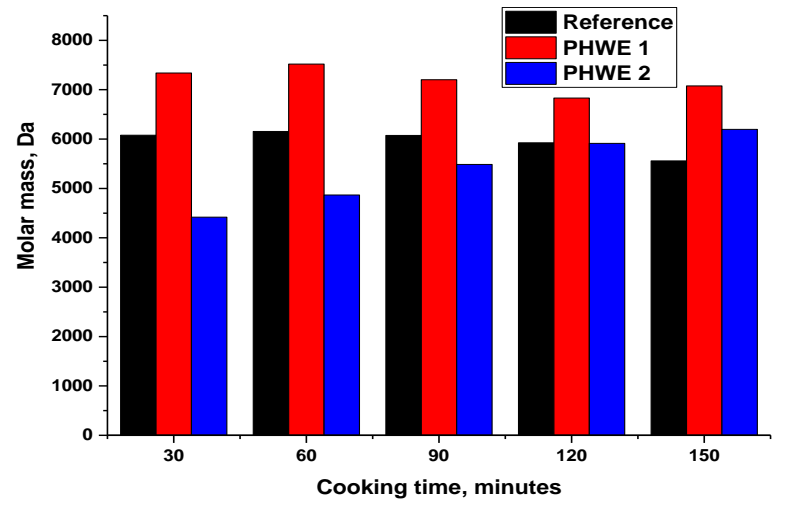

Fig $2-\overline{M_{w}}$ values of dissolved lignin in BLs from the soda-AQ cooking of untreated (Reference) and pre-treated (PHWE) spruce sawdust. 

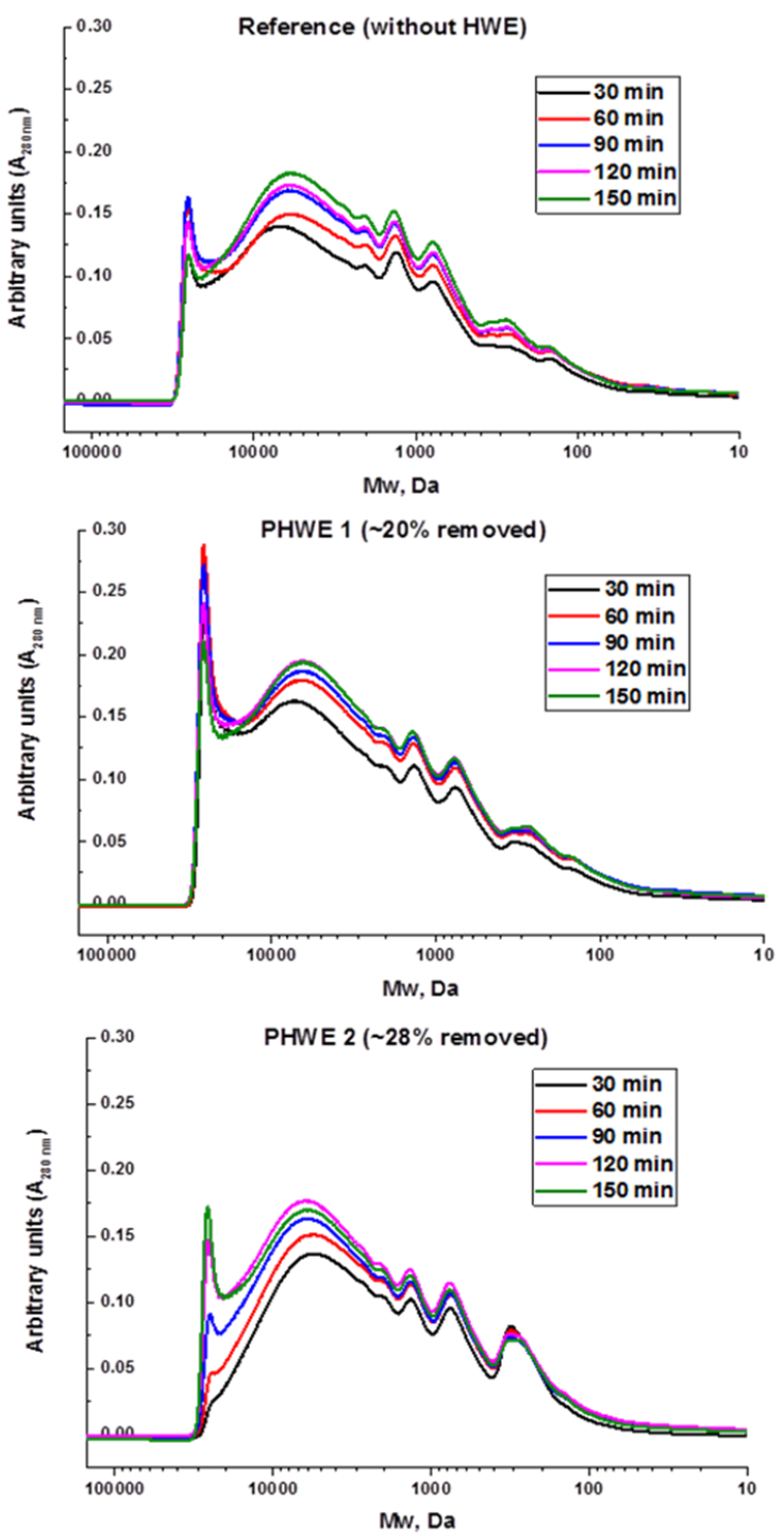

Fig 3 - MMDs of dissolved lignin in BLs from the soda-AQ pulping of untreated (Reference) and two differently pre-treated (HWE) spruce sawdust.

increased significantly, most likely due to the enhanced peeling reactions degrading wood carbohydrates (mainly cellulose).

In addition to the content of volatile acids, profound effects caused by the pre-treatment experiments conducted with varying intensities could be observed when the formation of hydroxy acids during the subsequent cooking experiments was evaluated (Fig 5 and Table 5). The harsh hot-water pre-treatment distinctly increased the formation of hydroxy acids during the cooking experiments. Applying moderate pre-treatment (PHWE 1) conditions caused an increase of $10-30 \%$ in hydroxy acid content, whereas an increase of 30-80\% was observed under harsh (PHWE 2) pre-treatment conditions prior to pulping. These results indicated that degradation reactions were clearly increased when autohydrolyzed wood was used for pulping. Due to the fact carbohydrate that after harsh autohydrolysis conditions (PHWE 2) the main part ( 95\%) of the

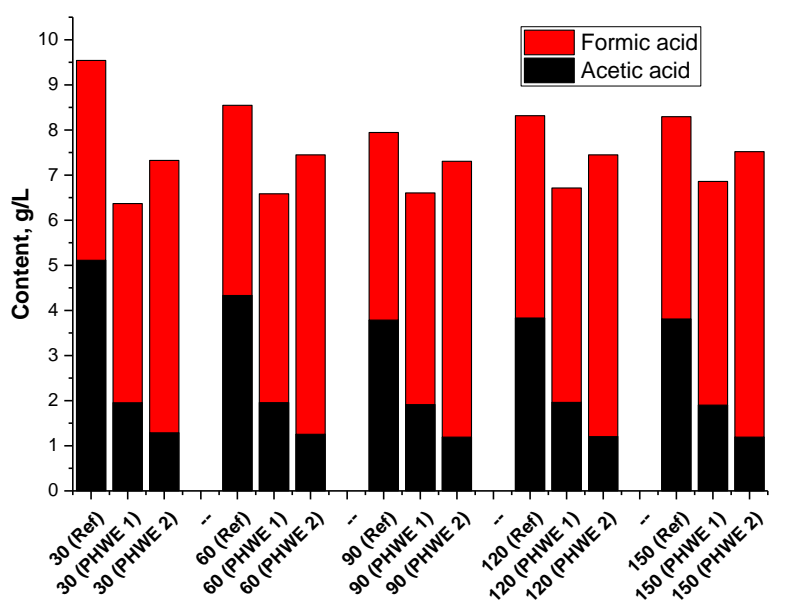

Fig 4 - Content of volatile acids in BLs from the soda-AQ pulping of untreated (reference) and differently pre-treated (PHWE) spruce sawdust.

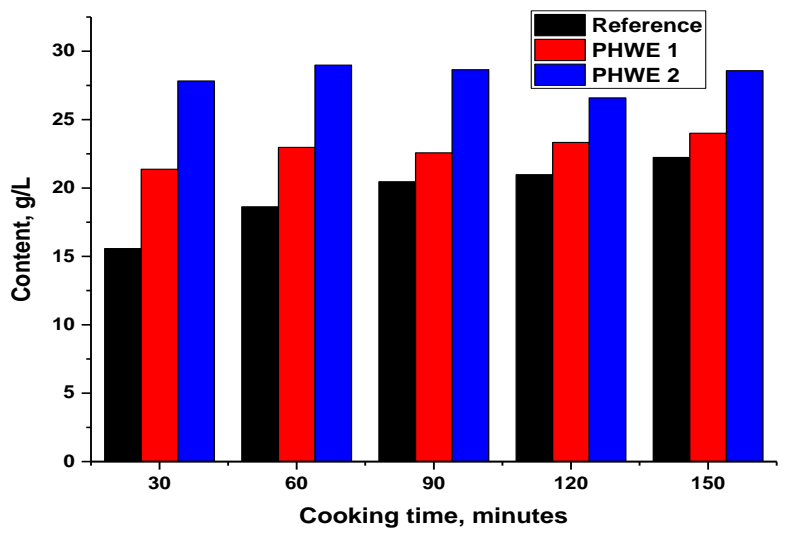

Fig 5 - Content of hydroxy acids in BLs from the soda-AQ pulping of untreated (reference) and differently pre-treated (PHWE) spruce sawdust.

Table 5 - Contents of some selected acids in BLs (g/l)

\begin{tabular}{lccccc}
\hline Time/min & $\mathbf{3 0}$ & $\mathbf{6 0}$ & $\mathbf{9 0}$ & $\mathbf{1 2 0}$ & $\mathbf{1 5 0}$ \\
\hline Reference & 15.6 & 18.6 & 20.5 & 21.0 & 22.2 \\
Low-MM $^{*}$ & 3.7 & 4.7 & 5.5 & 5.9 & 6.2 \\
Xyloisosaccharinic & 0.6 & 0.6 & 0.7 & 0.7 & 0.8 \\
Glucoisosaccharinic $^{* *}$ & 5.7 & 7.0 & 7.6 & 7.6 & 7.9 \\
Others & 5.5 & 6.2 & 6.5 & 6.8 & 7.3 \\
\hline PHWE 1 $^{*}$ & 21.4 & 23.0 & 22.6 & 23.3 & 24.0 \\
Low-MM $^{*}$ & 4.7 & 5.7 & 5.8 & 6.2 & 6.7 \\
Xyloisosaccharinic & 2.0 & 2.0 & 2.0 & 2.0 & 2.0 \\
Glucoisosaccharinic $^{* *}$ & 7.4 & 7.7 & 7.6 & 7.8 & 7.8 \\
Others & 7.3 & 7.6 & 7.3 & 7.3 & 7.6 \\
\hline PHWE 2 & 27.8 & 29.0 & 28.7 & 26.6 & 28.6 \\
Low-MM & 7.2 & 7.7 & 7.8 & 7.3 & 8.1 \\
Xyloisosaccharinic & 1.6 & 1.7 & 1.7 & 1.5 & 1.6 \\
Glucoisosaccharinic** & 10.5 & 11.1 & 10.8 & 10.0 & 10.6 \\
Others & 8.4 & 8.5 & 8.4 & 7.8 & 8.3 \\
\hline Glyclicand & & &
\end{tabular}

*Glycolic and lactic acids, MM refers to molar mass.

**Includes $\alpha$ - and $\beta$-glucoisosaccharinic acids.

carbohydrate fraction was composed of glucose (i.e., cellulosic fiber), it could be concluded that severe degradation of the fiber itself must have taken place during harsh autohydrolysis combined with pulping. 
Based on the data presented in Fig 5 and Table 5, a significant portion of cooked materials was dissolved as hydroxy acids. From PHWE 2 material, $21 \%-27 \%$ of the material loaded into the reactors (from $15 \%$ to $19 \%$ of the o.d. initial feedstock) dissolved as hydroxy acids. Corresponding values for PHWE 1 and reference materials were $17 \%-27 \%(13 \%-15 \%$ of the initial o.d. feedstock) and $13 \%-23 \%$, respectively. Of the individual hydroxy acids, especially the contents of glucoisosaccharinic acids were clearly increased, thus suggesting especially the partial degradation of glucose moieties in cellulose.

\section{Conclusions}

In this study, the main aim was to determine the effects of hot-water pre-treatments (conducted with different intensities) on the subsequent sulfur-free pulping of softwood sawdust and on the formation of the two main degradation product groups, lignin and organic acids (volatile and hydroxy acids). It could be concluded that the moderately performed PHWE enhanced the dissolution of lignin out from the wood matrix, thus creating potential lignin fractions for further utilization, for example, in polymeric applications. However, when drastically changing the pre-treatment conditions to harsher states, the feedstock material became profoundly resistant to alkaline sulfur-free delignification. This phenomenon could be observed in several ways. When compared to the reference materials (untreated and moderately pre-treated sawdust), the severely pre-treated material was very poorly defibrated. In addition, less and smaller-molar-mass lignin was dissolved under the same cooking conditions from material pre-treated harshly. Possible reasons for such a behavior could include, for example, various condensation reactions taking place in the lignin structure under harsh pre-treatment conditions, which rendered the lignin resistant to further delignification.

\section{Acknowledgements}

Financial support within the framework of the Future Biorefinery Joint Research 2 (FuBio JR 2) Research Program (Finnish Bioeconomy Cluster, FIBIC), Fortum Foundation, and Maj and Tor Nessling Foundation is gratefully acknowledged.

\section{Literature}

Adams, T.N. (1997): General characteristics of kraft black liquor recovery boilers, In: Adams, T.N. (ed.) Kraft Recovery BoilersTappi, Press, Atlanta, GA, USA, pp. 3-38.

Al-Dajani, W.W. and Tschirner, U.W. (2008): Pre-extraction of hemicelluloses and subsequent kraft pulping Part I: alkaline extraction, Tappi J., 7(5), 3-8.

Alén, R. (2000): Basic chemistry of wood delignification, In: Stenius, P. (ed.), Forest Products Chemistry, Fapet Oy, Helsinki, Finland, pp. 58-104.

Alén, R. (2011): Principles of biorefining, In: Alén, R. (ed.), Biorefining of Forest Resources, Paper Engineers' Association/Paperi ja Puu Oy, Helsinki, Finland, pp. 56-115.

Alén, R. and Hartus, T. (1988): UV spectrophotometric determination of lignin from alkaline pulping liquors, Cellul. Chem. Technol., 22(6), 613-618.
Alén, R., Jännäri, P. and Sjöström, E. (1985): Gas-liquid chromatographic determination of volatile fatty acids $\mathrm{C}_{1}-\mathrm{C}_{6}$, and lactic acid as their benzyl esters on a fused-silica capillary column, Finn. Chem. Lett., 12(5), 190-192.

Alén, R., Niemelä, K. and Sjöström, E. (1984): Gas-liquid chromatographic separation of hydroxy monocarboxylic acids and dicarboxylic acids on a fused-silica capillary column, J. Chromatogr., 301, 273-276.

Amidon, T.E. and Liu, S. (2009): Water-based woody biorefinery, Biotechnol. Adv., 27(5), 542-550.

Baijpai, P. (2012): Integrated forest biorefinery, In: Bajpai, P. (ed.), Biotechnology for Pulp and Paper Processing, Springer, New York, NY, USA, pp. 375-402.

Borrega, M., Nieminen, K. and Sixta, H. (2011). Effects of hot water extraction in a batch reactor on the delignification of birch wood, BioRes. 6(2), 1890-1903.

Brasch, D.J. and Free, K.W. (1965): Prehydrolysis-kraft pulping of Pinus radiata grown in New Zealand, Tappi J., 48(4), 245-248.

Carvalheiro, F., Duarte, L.C. and Gírio, F.M. (2008): Hemicellulose biorefineries: a review on biomass pretreatments, J. Sci. Ind. Res., 67(11), 849-864.

Chen, X., Lawoko, M. and van Heiningen, A. (2010): Kinetics and mechanism of autohydrolysis of hardwoods, Biores. Technol., 101(20), 7812-7819.

Chua, M.G.S. and Wayman, M. (1979): Characterization of autohydrolysis aspen (P. tremuloides) lignins. Part 1. Composition and molecular weight distribution of extracted autohydrolysis lignin, Can. J. Chem., 57(10), 1141-1149.

Garrote, G., Domínguez, H. and Parajó, J.C. (1999a): Hydrothermal processing of lignocellulosic materials, Holz als Roh- und Werkst., 57(3), 191-202.

Garrote, G., Domínguez, H. and Parajó, J.C. (1999b): Mild autohydrolysis: an environmentally friendly technology for xylooligosaccharide production from wood, J. Chem. Technol. Biotechnol., 74(11), 1101-1109.

Garrote, G., Domínguez, H. and Parajó, J.C. (2001): Study on the deacetylation of hemicelluloses during hydrothermal processing of Eucalyptus wood, Holz als Roh- und Werkst., 59(1), 53-59.

Hendriks, A.T.W.M. and Zeeman, G. (2009): Pretreatments to enhance the digestibility of lignocellulosic biomass, Biores. Technol., 100(1), 10-18.

Käkölä, J.M., Alén, R.J., Isoaho, J.P. and Matilainen, R.B. (2008): Determination of low-molecular-mass aliphatic carboxylic acids and inorganic anions from kraft black liquors by ion chromatography, J. Chromatogr. A, 1190(1-2), 150-156.

Käkölä, J., Alén, R., Pakkanen, H., Matilainen, R. and Lahti, K. (2007): Quantitative determination of the main aliphatic carboxylic acids in wood kraft black liquors by high-performance liquid chromatography-mass spectrometry, J. Chromatogr. A, 1139(2), 263-270.

Kamm, B. and Kamm, M. (2004): Principles of biorefineries, Appl. Microbiol. Biotechnol., 64(2), 137-145.

Kamm, B., Kamm, M., Gruber, P.R. and Kromus, S. (2006): Biorefinery systems - An overview, In: Kamm, B., Gruber, P.R. and Kamm, M. (eds.), Biorefineries - Industrial Processes and 
Products, Status Quo and Future Directions, Vol. 1, Wiley-VCH Verlag GmbH \& Co. KGaA, Weinheim, Germany, pp. 3-40.

Kumar, P., Barret, D.M., Delwiche, M.J. and Stroeve, P. (2009): Methods for pretreatment of lignocellulosic biomass for efficient hydrolysis and biofuel production, Ind. Eng. Chem. Res., 48(8), 3713-3729.

Lehto, J., Pakkanen, H. and Alén, R. (2015): Molecular mass distribution of sulfur-free lignin from alkaline pulping preceded by hot-water-extraction, Appita J., 68(2), 149-158.

Leschinsky, M., Weber, H.K., Patt, R. and Sixta, H. (2009): Formation of insoluble components during autohydrolysis of Eucalyptus globulus, Lenzing. Ber., 87, 16-25.

Leschinsky, M., Zuckerstätter, G., Weber, H.K., Patt, R. and Sixta, H. (2008): Effect of autohydrolysis of Eucalyptus globulus wood on lignin structure. Part 2: Influence of autohydrolysis intensity, Holzforschung, 62(6), 653-658.

Li, J. and Gellerstedt, G. (2008): Improved lignin properties and selectivity by modifications in the autohydrolysis process of aspen wood, Ind. Crops Prod., 27(2), 175-181.

Niemelä, K. (1988a): GLC-MS studies on pine kraft black liquors, Part I. Identification of monomeric compounds, Holzforschung, 42(3), 169-173.

Niemelä, K. (1988b): GLC-MS studies on pine kraft black liquors, Part II. Identification of hydroxy acids with a stilbene structure, Holzforschung, 42(3), 175-176.

Niemelä, K. (1989): GLC-MS studies on pine kraft black liquors Part V. Identification of catechol compounds, Holzforschung, 43(2), 99-103.

Niemelä, K. (1990a): Conversion of xylan, starch, and chitin into carboxylic acids by treatment with alkali, Carbohydr. Res., 204, 37-49.

Niemelä, K. (1990b): The formation of hydroxy monocarboxylic acids and dicarboxylic acids by alkaline thermochemical degradation of cellulose, J. Chem. Techn. Biotechnol., 48(1), 17-28.

Niemelä, K., Alén, R. and Sjöström, E. (1985): The formation of carboxylic acids during kraft and kraft-anthraquinone pulping of birch wood, Holzforschung, 39(3), 167-172.

Niemelä, K. and Sjöström, E. (1986a): Alkaline degradation of mannan, Holzforschung, 40(1), 9-14.

Niemelä, K. and Sjöström, E. (1986b): Simultaneous identification of aromatic and aliphatic low molecular weight compounds from alkaline pulping liquor by capillary gas-liquid chromatography-mass spectrometry, Holzforschung, 40(6), 361-368.

Niemelä, K. and Sjöström, E. (1986c): The conversion of cellulose into carboxylic acids by a drastic alkali treatment, Biomass, 11(3), 215-221.

Pakkanen, H. and Alén, R. (2012): Molecular mass distribution of lignin from the alkaline pulping of hardwood, softwood, and wheat straw, J. Wood Chem. Technol., 32(4), 279-293.

Pakkanen, H. and Alén, R. (2013): Alkali consumption of aliphatic carboxylic acids during alkaline pulping of wood and nonwood feedstocks, Holzforschung, 67(6), 643-650.

Pakkanen, H., Paloheimo, T. and Alén, R. (2012): Characterization of dissolved material during the initial phase of softwood kraft pulping, Tappi J., 11(1), 35-43.
Penttilä, P.A., Kilpeläinen, P., Tolonen, L., Suuronen, J.-P., Sixta, H., Willför, S. and Serimaa, R. (2013): Effects of pressurized hot water extraction on the nanoscale structure of birch sawdust, Cellulose, 20(5), 2335-2347.

Ristolainen, M., Alén, R. and Knuutinen, J. (1996): Characterization of TCF effluents from kraft pulp bleaching. I. Fractionation of hardwood lignin-derived material by GPC and UF, Holzforschung, 50(1), 91-96.

Sánchez, O.J. and Cardona, C.A. (2008): Trends in biotechnological production of fuel ethanol from different feedstocks, Biores. Technol., 99(13), 5270-5295.

Santos, R.B., Hart, P.W., Jameel, H. and Chang, H.-M. (2013): Wood based lignin reactions important to the biorefinery and pulp and paper industries, BioRes., 8(1), 1456-1477.

Schild, G., Sixta, H. and Testova, L. (2010): Multifunctional alkaline pulping, delignification and hemicellulose extraction, Cellul. Chem. Technol., 44(1-3), 35-45.

Sixta, H., Potthast, A. and Krotschek, A.W. (2006): Chemical pulping processes, In: Sixta, H. (ed.) Handbook of Pulp, WileyVCH Verlag GmbH\&Co, Weinheim, Germany, pp. 109-510.

Sixta, H. and Schild, G. (2009): A new generation kraft process, Lenzing. Ber., 87, 26-37.

Smith, R.M. (2002): Extractions with superheated water, J. Chromatogr. A., 975(1), 31-46.

Swan, B. (1965): Isolation of acid-soluble lignin from the Klason lignin determination, Svensk Papperstidning, 68, 791-795.

Teo, C.C., Tan, S.N., Yong, J.W.H., Hew, C.S. and Ong, E.S. (2010): Pressurized hot water extraction (PHWE), J. Chromatogr. A, 1217(16), 2484-2494.

Timilsena, Y.P., Audu, I.G., Rakshit, S.K. and Brosse, N. (2013): Impact of the lignin structure of three lignocellulosic feedstocks on their organosolv delignification. Effect of carbonium ion scavengers, Biom. Bioen., 52, 151-158.

Tsutsumi, Y., Kondo, R., Sakai, K. and Imanura, H. (1995): Difference in reactivity between syringyl and guaiacyl lignin in alkaline systems, Holzforschung, 49(5), 423-428.

Tunc, M.S. and van Heiningen, A.R.P. (2009): Autohydrolysis of mixed southern hardwoods: Effect of P-factor, Nord. Pulp Pap. Res. J., 24(1), 46-51.

Vakkilainen, E. (2008): Chemical recovery, In: Tikka, P. (ed.), Chemical Pulping, Part 2, Recovery of Chemicals and Energy, Paper Engineers' Association, Helsinki, Finland, pp. 10-35.

van Heiningen, A. (2006): Converting a kraft pulp mill into an integrated forest biorefinery, Pulp Paper Can., 107(6), 38-43.

Wiboonsirikul, J. and Adachi, S. (2008): Extraction of functional substances from agricultural products or by-products by subcritical water treatment, Food Sci. Technol. Res., 14(4), 319-328.

Zhu, M.-Q., Wen, J.-L., Su, Y.-Q., Wei, Q. and Sun, R.-C. (2015): Effect of structural changes if lignin during the autohydrolysis and organosolv pretreatment on Eucommia ulmoides Oliver for an effective enzymatic hydrolysis, Biores. Technol., 185,378-385.

Manuscript received October 8, 2015 Accepted December 21, 2015 